\title{
Noise Reduction Multi-Carrier Differential Chaos Shift Keying System*
}

\author{
Hong Min Zhou ${ }^{\dagger}$ \\ Nanjing University of Posts and Telecommunications, \\ Nanjing, P. R. China \\ †zhouhm@njupt.edu.cn \\ Ying Zhang* and Ying $\mathrm{Yu}^{\S}$ \\ College of Electronic and Optical Engineering $\&$ \\ College of Microelectronics, \\ Nanjing University of Posts and Telecommunications, \\ Nanjing, P. R.China \\ tzhangying@njupt.edu.cn \\ §ying_yu_001@163.com \\ Received 10 November 2017 \\ Accepted 1 April 2018 \\ Published 4 May 2018
}

\begin{abstract}
In multi-carrier differential chaos shift keying (MC-DCSK) system, channel noises pollute both the reference and data signals, resulting in deteriorated performance. To reduce noises in received signals in MC-DCSK, a novel noise reduction MC-DCSK (NR-MC-DCSK) system is proposed in this paper. The proposed system utilizes duplicated chaotic samples, rather than different ones, as the reference. At the receiver side, identical samples can be averaged before correlation detection, which helps decrease the noise interferences and thus brings performance improvement. Theoretical bit error rate (BER) expressions are derived and verified by simulation results for additive white Gaussian noise and multipath Rayleigh fading channels. Finally, comparisons to MC-DCSK and other DCSK-based systems are given to confirm the superiority of the proposed system in BER performance.
\end{abstract}

Keywords: Chaos; multi-carrier DCSK; noise reduction; bit error rate; multipath Rayleigh fading channel.

\footnotetext{
*This paper was recommended by Regional Editor Zoran Stamenkovic.

$\dagger$ Corresponding author.
}

This is an Open Access article published by World Scientific Publishing Company. It is distributed under the terms of the Creative Commons Attribution 4.0 (CC-BY) License. Further distribution of this work is permitted, provided the original work is properly cited. 


\section{Introduction}

With noise-like spectrum as well as sharp auto-correlation and low cross-correlation characteristics, chaotic signals have been applied in wireless communications in recent years. ${ }^{1-5}$ As low-cost substitutions for traditional spread spectrum (SS) systems, lots of chaos-based modulation schemes that map data symbols to nonperiodic and wideband chaotic wavelets have been proposed and studied. ${ }^{6-12}$ They not only have similar advantages as other SS communication systems, including antijamming capability and resistance to multipath fading, but also achieve better data security. ${ }^{13}$

Due to simple circuits, differential chaos shift keying (DCSK) in Ref. 6 becomes one of the most popular chaos-based modulation systems. To avoid the requirement of chaos synchronization and channel estimation at the receiver side, DCSK transmits the reference and data signals in the first and second halves of one bit duration, respectively. However, DCSK suffers from low attainable bit rate and low spectrum efficiency as one half of bit duration and energy are devoted to reference transmission. To increase bit rate and spectral efficiency, many efforts have been made and plenty of enhanced versions of DCSK systems have been designed. ${ }^{8-10}$ In Ref. 8 and 9, Hilbert transform and multiple time delays are adopted, respectively, to double the bit rate with respect to DCSK, but with almost no gain in BER performance. Similarly, the reference and data-bearing signals in DCSK are transmitted in a parallel way via the orthogonal Inphase and quadrature channels in Ref. 10 for doubled bit rate, which helps to remove the radio frequency delay lines that are quite difficult to integrate in ultrawide band (UWB) communications but brings no BER performance improvement in comparison with DCSK. For higher bit rate, authors in Refs. 11 and 12 employ, respectively, Walsh codes and chaotic codes to separate the reference and multiple data-bearing signals for simultaneous transmission, resulting in complicated system design. For the same purpose, a short chaotic reference signal has been utilized in Ref. 14 and applied to simultaneous wireless information and power transfer in Ref. 15, which not only helps to increase the energy efficiency but also improve the bit error rate (BER) performance.

As an effective way to combat with the hostile propagation environments in high bit rate mobile communications, multi-carrier modulation (MCM) technique has recently been applied into chaos-based modulations for the purpose of improving performances. In 2013, a multi-carrier DCSK (MC-DCSK) system is proposed in Ref. 16 by combining MCM with DCSK based on FDMA. This system converts a serial high rate bit stream into multiple parallel low rate bit sub-streams and sends the reference and multiple data signals, respectively, over one predefined subcarrier and the other remaining subcarriers. In 2015, MC-DCSK system has been extended into the cognitive multi-user access scenario, ${ }^{17}$ and thus an analog network coding scheme is designed. In 2016, a multi-user MC-DCSK system, orthogonal frequency division multiplexing DCSK (OFDM-DCSK), was proposed in Ref. 18, where all 
users share some predefined subcarriers for data transmission. To further increase the spectral efficiency, MCM is applied in multi-level chaos shift keying (CSK) modulation, and a novel multi-carrier CSK (MC-CSK) modulation system is proposed in Ref. 19. Compared to DCSK, MCM not only help these systems solve the delay line problem but also brings them higher bit rate, higher spectral efficiency, increased energy efficiency and better BER performance. ${ }^{16-19}$ However, all of these systems still suffer from a major drawback that both the received reference and data signals are polluted by the channel noise, which will greatly degrade the system BER performance.

In this paper, a novel noise reduction MC-DCSK (NR-MC-DCSK) modulation system is proposed, in which duplicated chaotic samples are used as the message bearers. By averaging redundant samples at the receiver, noise interferences in both the reference and data signals can be reduced before correlation, leading to a remarkable gain in BER performance. Under the multipath Rayleigh fading channel, the BER performance of the proposed system is analyzed and validated by the simulation results. Performance comparisons are also made to other DCSK systems, including MC-DCSK, NR-DCSK in Ref. 20 and DCSK. Both the theoretical and simulation results show that the proposed system performs much better than the other MC-DCSK systems without any performance loss in bit rate and energy efficiency.

The rest of the paper is organized as follows. In Sec. 2, the basis of MC-DCSK system is briefly reviewed. In Sec. 3, the basic scheme of NR-MC-DCSK system is described. In Sec. 4, the BER performances of the NR-MC-DCSK system over AWGN and multipath Rayleigh fading channels are derived. Simulation results are reported with comparisons in Sec. 5. Finally, conclusions are given in Sec. 6 .

\section{Brief Review of MC-DCSK System}

For higher bit rate and better energy efficiency, MC-DCSK system converts a serial high rate bit stream into $M-1$ parallel low rate bit sub-streams. In one symbol duration, data bits in all sub-streams are spread due to multiplication in time with current chaotic reference signal.

The chaotic reference signal varies from symbol to symbol. For one isolated symbol duration, a certain chaotic map $x_{i+1}=f\left(x_{i}\right)$ outputs a chaotic sequence with $\beta$ different samples. Based on these samples, current reference signal $x(t)$ can be obtained as

$$
x(t)=\sum_{i=0}^{\beta-1} x_{i} h_{T}\left(t-i T_{c}\right) .
$$

Here, $x_{i}$ is the $i$ th sample of the chaotic sequence, and $h_{T}(t)$ is the impulse response of a square-root-raised cosine filter with duration of $T_{c}$, normalized energy and a roll-off 
factor of $\alpha$. To satisfy the Nyquist criterion, the frequency spectrum of $h_{T}(t)$ has been limited to $\left[-B_{c} / 2, B_{c} / 2\right]$ with $B_{c}=(1+\alpha) / T_{c}$.

To avoid channel estimation and chaos synchronization, MC-DCSK system modulates the chaotic signal in (1) onto the first subcarrier $f_{0}$ as the reference, while the other subcarriers $f_{1}, \ldots, f_{M-1}$ are allocated to $M-1$ bit sub-streams. Thus, the transmitted signal of MC-DCSK in current symbol duration is represented as

$$
s(t)=x(t) \cos \left(2 \pi f_{0} t+\theta_{0}\right)+\sum_{m=1}^{M-1} d_{m} x(t) \cos \left(2 \pi f_{m} t+\theta_{m}\right) .
$$

In which, $\theta_{m}$ is phase angle introduced in the carrier modulation process, and $d_{m}$ is the data bit carried by the $m$ th subcarrier.

With assumptions that the received signal is only corrupted by an AWGN noise with PSD of $N_{0} / 2$, the reference samples and the data samples can be retrieved from $M$ subcarriers, and they can be, respectively, denoted as

$$
\begin{gathered}
r_{i}=x_{i}+n_{i, 0}, \\
y_{i, m}=d_{m} x_{i}+n_{i, m}, \quad m=1, \ldots, M-1 .
\end{gathered}
$$

Here, $n_{i, m}$ is the $i$ th sample of the noise that affects the signal transmitted on the $m$ th subcarrier.

By correlating the reference $r_{i}$ and the data-bearing signal $y_{i, m}$ over one symbol duration, data bit $d_{m}$ can be recovered as

$$
\tilde{d}_{m}=\operatorname{sign}\left[\sum_{i=0}^{\beta-1} r_{i} y_{i, m}\right], \quad m=1, \ldots, M-1,
$$

where sign $[\cdot]$ is the sign function.

According to (3) and (4), both the reference and data-bearing signals are contaminated by the channel noise, which increases the uncertainty in decision variables in (5) and eventually leads to degraded BER performance of MC-DCSK.

\section{Noise Reduction MC-DCSK System}

In the proposed system, the spreading sequence is generated based on repeated chaotic samples rather than different ones. As illustrated in Fig. 1, in one isolated symbol duration, the chaotic generator outputs $N$ chaotic samples $\left\{x_{i} \mid i=\right.$ $0, \ldots, N-1\}$, and each sample is repeated for $R$ times and then saved in the buffer to form the spreading sequence $\left\{\hat{x}_{j} \mid j=0, \ldots, N R-1\right\}$. It is remarked that this design of introducing redundancy into the frame helps to improve the performance of the system, as the introduced redundancy can be utilized and averaged at the receiver side. 


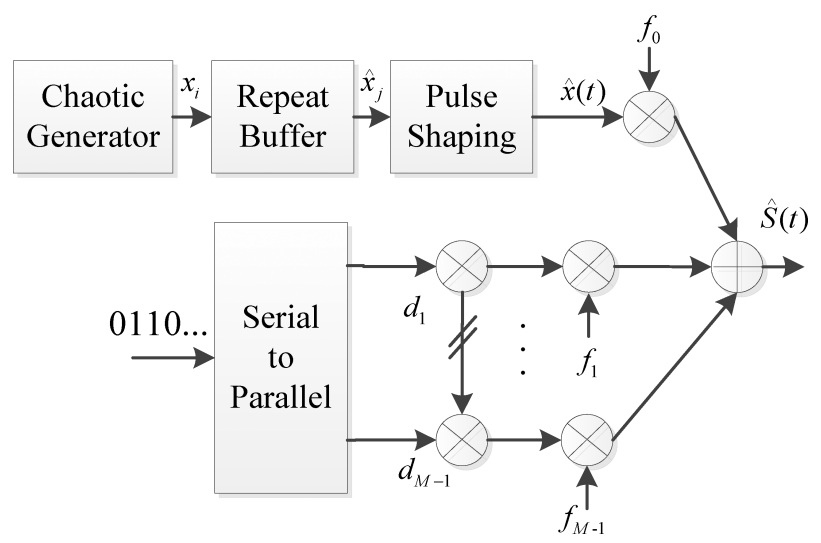

Fig. 1. The block diagram of NR-MC-DCSK transmitter.

During this same symbol period, the samples stored in the repeat buffer in Fig. 1 satisfy

$$
\hat{x}_{j}=x_{j \% R}, \quad j=0, \ldots, N R-1,
$$

where $\%$ is the ceiling operator.

Accordingly, the current reference signal in the proposed system can be denoted as

$$
\hat{x}(t)=\sum_{j=0}^{N R-1} \hat{x}_{j} h_{T}\left(t-j T_{c}\right) .
$$

For simplicity, NR-MC-DCSK adopts the same multi-carrier transmission mechanism and subcarrier allocation method given in Ref. 16. In the first subcarrier, current reference signal in (7) is transmitted. All data bits in the $M-1$ parallel low rate bit sub-streams are carried by the other $M-1$ subcarriers, and each data bit is modulated onto the reference signal by multiplication. As the sum of signals transmitted in all subcarrier channels, the transmitted signal of NR-MC-DCSK can be represented as

$$
\hat{S}(t)=\hat{x}(t) \cos \left(2 \pi f_{0} t+\theta_{0}\right)+\sum_{m=1}^{M-1} d_{m} \hat{x}(t) \cos \left(2 \pi f_{m} t+\theta_{m}\right) .
$$

In the receiver given in Fig. 2, the received signal $\hat{r}(t)$ first is multiplied with the synchronized carriers of the $M$ subcarriers, respectively. The resultant signals are then fed into $M$ identical filters matched to the pulse signal $h_{T}(t)$ separately so that the peak output signal-to-noise ratio can be maximized at each sampling time $t=j T_{c}$. We assume that the received signal is only corrupted by an additive Gaussian white noise (AWGN) $n(t)$ with zero mean and a variance of $N_{0} / 2$. 


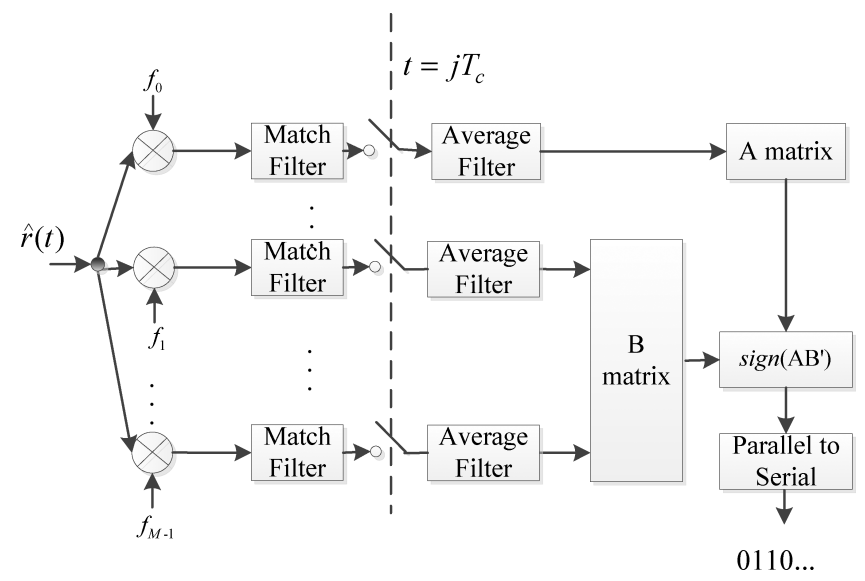

Fig. 2. The structure of the receiver in NR-MC-DCSK system.

The output of the $m$ th sampler in Fig. 2 at the time of $t=j T_{c}$ is obtained as

$$
c_{j, m}=\left\{\begin{array}{ll}
\hat{x}_{j}+n_{j, m}, & m=0 \\
d_{m} \hat{x}_{j}+n_{j, m} & m=1, \ldots, M-1
\end{array} \quad \text { for } j=0, \ldots, N R-1,\right.
$$

where $n_{j, m}$ is the $j$ th sample of the noise that affects the signal transmitted on the $m$ th subcarrier.

Later, samples in each branch are averaged using a moving average filter with a window size of $R$. Based on the relationship in (6), the output of the $m$ th average filter can be represented as

$$
\bar{c}_{i, m}=\left\{\begin{array}{ll}
x_{i}+\varepsilon_{i, m}, & m=0 \\
d_{m} x_{i}+\varepsilon_{i, m}, & m=1, \ldots, M-1
\end{array} \text { for } i=0, \ldots, N-1\right.
$$

with

$$
\varepsilon_{i, m}=\frac{1}{R} \sum_{j=(i-1) R}^{i R-1} n_{j, m}
$$

Considering that the noise samples sampled at different times are independent and identically distributed random variables, using the moving average filtering operation at the receiver side can help reduce the noise variance in (10) by $R$ times in comparison with (5), which results in a significant enhancement of the NR-MCDCSK performance.

Finally, the averaged samples carrying data are correlated with the averaged reference samples. And the data bit carried by the $m$ th subcarrier can be estimated 
by the following decision rule:

$$
\tilde{d}_{m}=\operatorname{sign}\left[\sum_{i=0}^{N-1} \bar{c}_{i, m} \bar{c}_{i, 0}\right], \quad m=1, \ldots, M-1 .
$$

According to Ref. 16, energy efficiency of the proposed system can be evaluated by the Data-energy-to-Bit-energy Ratio (DBR), which is represented as

$$
\mathrm{DBR}=\frac{M-1}{M}
$$

DBR in (3) happens to be identical to the DBR of MC-DCSK derived in Ref. 16. This means that NR-MC-DCSK obtains same energy efficiency in comparison with MC-DCSK with the same number of subcarriers. Besides, as both NR-MC-DCSK and MC-DCSK systems transmit $M-1$ bits in each symbol duration, these two systems obtains the same bit rate if same number of subcarriers and same spreading factor are used.

\section{Performance Analysis}

In this section, the analytical expressions for the noise performance of the proposed system are derived over AWGN and multipath Rayleigh fading channels, respectively. Here, the logistic map $x_{i+1}=1-2 x_{i}^{2}$ in Ref. 13 is used in the chaotic generator in Fig. 1. The output sample of this logistic map is normalized to have a mean value of zero and a variance of one. Besides, a high spreading factor is assumed throughout this paper, hence, the bit energy can be regarded as constant. ${ }^{21}$

\subsection{AWGN channel}

Substituting (10) into (12), the decision variable for bit $d_{m}$ transmitted over the $m$ th subcarrier can be written as

$$
Z_{m}=\sum_{i=0}^{N-1}\left(x_{i}+\varepsilon_{i, 0}\right)\left(d_{m} x_{i}+\varepsilon_{i, m}\right) .
$$

Assuming $d_{m}=+1$, the expectation and the variance of the decision variable in (14) can be derived as

$$
\begin{gathered}
E\left[Z_{m} \mid d_{m}=+1\right]=\frac{2(M-1)}{M R} E_{b}, \\
\operatorname{var}\left[Z_{m} \mid d_{m}=+1\right]=\frac{4(M-1)}{M R^{2}} E_{b} N_{0}+\frac{N}{R^{2}} N_{0}^{2},
\end{gathered}
$$

where $E_{b}$ is the bit energy of the transmitted signal, $E[\cdot]$ denotes the expectation operator, and $\operatorname{var}[\cdot]$ is the variance operator. 
According to the analysis in Refs. 21 and 22, the correlation output in (14) are effectively Gaussian distributed. If the transmitted bits are equally probable, the BER of NR-MC-DCSK system is

$$
\begin{aligned}
\operatorname{BER}_{\mathrm{AWGN}} & =\frac{1}{2} \operatorname{prob}\left(Z_{m}>0 \mid d_{m}=-1\right)+\frac{1}{2} \operatorname{prob}\left(Z_{m}<0 \mid d_{m}=+1\right) \\
& =\frac{1}{2} \operatorname{erfc}\left(\frac{E\left[Z_{m} \mid d_{m}=+1\right]}{\sqrt{2 \operatorname{var}\left[Z_{m} \mid d_{m}=+1\right]}}\right) \\
& =\frac{1}{2} \operatorname{erfc}\left(\left(\frac{2 M}{(M-1)} \frac{N_{0}}{E_{b}}+\frac{\beta M^{2}}{2 R(M-1)^{2}}\left(\frac{N_{0}}{E_{b}}\right)^{2}\right)^{-\frac{1}{2}}\right),
\end{aligned}
$$

with $\operatorname{erfc}(\cdot)$ denotes the complementary error function ${ }^{23}$ and $\beta=N R$ denotes the spreading factor. For $R=1,(17)$ simplifies to the BER expression for MC-DCSK in AWGN channel in Ref. 16.

\subsection{Multipath Rayleigh fading channel}

Here, we consider a multipath Rayleigh fading channel model shown in Fig. 3, which consists of $L$ independent Rayleigh channels and channel coefficients of all paths are considered as unchanged during one certain symbol duration. This model is commonly used in the analysis of spread-spectrum wireless communication systems. ${ }^{16-19}$

The output of such an $L$-ray Rayleigh slow-fading channel is given by

$$
\hat{r}(t)=\sum_{l=1}^{L} \alpha_{l} \hat{S}\left(t-\tau_{l}\right)+n(t),
$$

where $\tau_{l}=\lambda_{l} T_{c}$ is the time delay of the $l$ th path and $\alpha_{l}$ is the propagation gain of the lth path. The probability density function of the channel coefficient in each path can be defined by

$$
f_{\alpha}(z)=\frac{z}{E\left[\alpha^{2}\right]} e^{-\frac{z^{2}}{2 E\left[\alpha^{2}\right]}}
$$

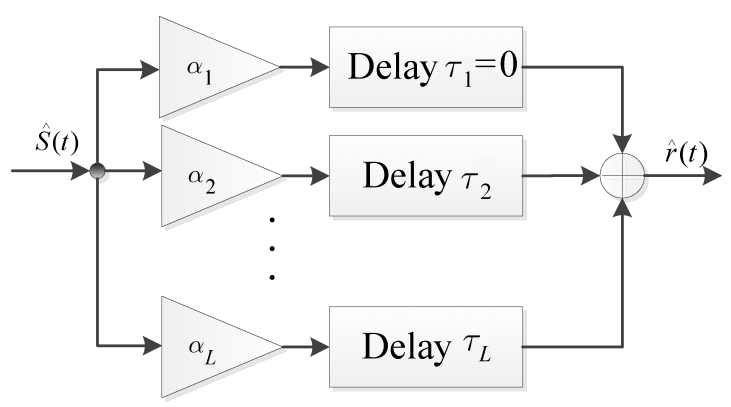

Fig. 3. Model of $L$-ray Rayleigh slow-fading channel. 
In this case, the output of the $m$ th average filter in Fig. 2 can be rewritten as

$$
\bar{c}_{i, m}= \begin{cases}\sum_{\substack{l=1 \\ i-\lambda_{l} \geq 0}}^{L} \alpha_{l} x_{i-\lambda_{l}}+\underbrace{L}_{\substack{\sum_{i=1} \alpha_{l} \alpha_{l}<0 \\ i-\lambda_{l}<0}} \alpha_{i-\lambda_{l}-N}+\varepsilon_{i, m}, & m=0 \\ \sum_{\substack{l=1 \\ i-\lambda_{l} \geq 0}}^{L} \alpha_{l} d_{m} x_{i-\lambda_{l}}+\underbrace{\sum_{\substack{l=1 \\ i-\lambda_{l}<0}}^{L} \alpha_{l} d_{m}^{\text {prev }} x_{i-\lambda_{l}-N}}_{\text {inter-symbol interference }}+\varepsilon_{i, m}, & m=1, \ldots, M-1 \\ & \text { for } i=0, \ldots, N-1 .\end{cases}
$$

with $d_{m}^{\text {prev }}$ represents the data bit transmitted over the $m$ th subcarrier in the previous symbol duration.

In our analysis, we assume that the path delay $\lambda_{l}$ is much smaller than the chaotic sample length $N$ i.e., $0<\lambda_{l} \ll N$, which is common in many practical applications. ${ }^{2,24}$ In this case, the inter-symbol interference (ISI) terms in (20) play a negligible role in correlation computation. By ignoring ISI, (20) can be approximated as follows:

$$
\bar{c}_{i, m}= \begin{cases}\sum_{l=1}^{L} \alpha_{l} x_{i-\lambda_{l}}+\varepsilon_{i, m}, & m=0 \\ d_{m} \sum_{l=1}^{L} \alpha_{l} x_{i-\lambda_{l}}+\varepsilon_{i, m}, & m=1, \ldots, M-1 .\end{cases}
$$

Based on (12), the decision variable for $d_{m}$ can be written as

$$
Z_{m}=\sum_{i=0}^{N-1}\left(\sum_{l=1}^{L} \alpha_{l} x_{i-\lambda_{l}}+\varepsilon_{i, 0}\right)\left(d_{m} \sum_{l=1}^{L} \alpha_{l} x_{i-\lambda_{l}}+\varepsilon_{i, m}\right) .
$$

For a given set of $\left(\alpha_{1}, \ldots, \alpha_{L}\right), Z_{m}$ in (22) follows a Gaussian distribution and the conditional BER of the proposed system can be obtained as

$$
\operatorname{BER}\left(\gamma_{b}\right)=\frac{1}{2} \operatorname{erfc}\left(\left(\frac{2 M}{(M-1)} \gamma_{b}^{-1}+\frac{\beta M^{2}}{2 R(M-1)^{2}} \gamma_{b}^{-2}\right)^{-\frac{1}{2}}\right),
$$

with $\gamma_{b}=\sum_{l=1}^{L} \alpha_{l}^{2} E_{b} / N_{0}$.

For the case of $L$ dissimilar propagation paths, the probability density function (PDF) of $\gamma_{b}$ has been derived in Ref. 23.

$$
p\left(\gamma_{b}\right)=\sum_{l=1}^{L} \frac{1}{\bar{\gamma}_{l}} \prod_{j=1, j \neq l}^{L} \frac{\bar{\gamma}_{l}}{\bar{\gamma}_{l}-\bar{\gamma}_{j}} e^{-\frac{\gamma_{b}}{\bar{\gamma}_{l}}}
$$

where $\bar{\gamma}_{l}=E\left[\alpha_{l}^{2} E_{b} / N_{0}\right]$. 
If the gains $\alpha_{l}$ of the propagation paths are identical, i.e., $\bar{\gamma}_{1}=\cdots=\bar{\gamma}_{L}=\bar{\gamma}$, the probability density function (PDF) of $\gamma_{b}$ can be calculated by

$$
p\left(\gamma_{b}\right)=\frac{\gamma_{b}^{L-1}}{(L-1) ! \bar{\gamma}^{L}} e^{-\frac{\gamma_{b}}{\gamma}} .
$$

The overall BER formula can be calculated by

$$
\operatorname{BER}_{\text {Rayleigh }}=\int \operatorname{BER}\left(\gamma_{b}\right) p\left(\gamma_{b}\right) d \gamma_{b}
$$

The lower bound of the integral given in formula (26) can be found in Ref. 25. Note that, with $L=1$ and $\gamma_{b}=E_{b} / N_{0}$, formula (26) turns into formula (17), which can also be used to evaluate the BER performance of NR-MC-DCSK over AWGN channel.

\section{Simulation Results}

In this section, the proposed system as well as MC-DCSK is simulated over AWGN and multipath Rayleigh fading channels. In these two systems, chaotic sequences are generated by the logistic map $x_{i+1}=1-2 x_{i}^{2}$. The roll-off factor $\alpha$ is set to 0.25 . The total bandwidth of all subcarriers is $4 \mathrm{MHz}$. For performance comparison, systems of NR-DCSK in Ref. 20 and DCSK using the same logistic map are also simulated.

To begin with, the simulated performances of NR-MCDCSK system are compared to the analytical ones calculated by (26) in Figs. 4 and 5 over AWGN and multipath Rayleigh fading channels, respectively. In this figure, one can observe that theoretical predictions match the simulated results quite well. This confirms the validity of our former analysis in Sec. 4. Besides, it is also observed in these two figures that the BER performance of NR-MC-DCSK improves with the increase of $R$. This can be explained by reduced variances of noises in (11).

The relationship between simulated BER and the repeat number $R$ is given in Fig. 6, in which the subcarrier number is set to 32 and the spreading factor is 300 . As shown in Figs. 4-6, the BER of the proposed system decreases with the repeat number $R$. This is due to reduced noise interference. Besides, it is also discovered in Fig. 6 that the BER improvement seems unobvious when $R$ is relatively large.

In the derivation of BER expression in Sec. 4, the effect of ISI is ignored, and thus (26) might be inaccurate for multipath Rayleigh fading channels with large time delays. To evaluate the effect of time delay on the system performance, Fig. 7 presents the simulated BERs as a function of the time delay of the second path in a tworay Rayleigh fading channel with identical average power gains. For all cases, it is assumed that $\lambda_{1}=0, R=2$ and $N=200$. In this figure, theoretical results are also given for comparison. Obviously, when the time delay is much smaller than the chaotic sample length, the simulated performances match the analytical ones quite 
Noise Reduction Multi-Carrier Differential Chaos Shift Keying System

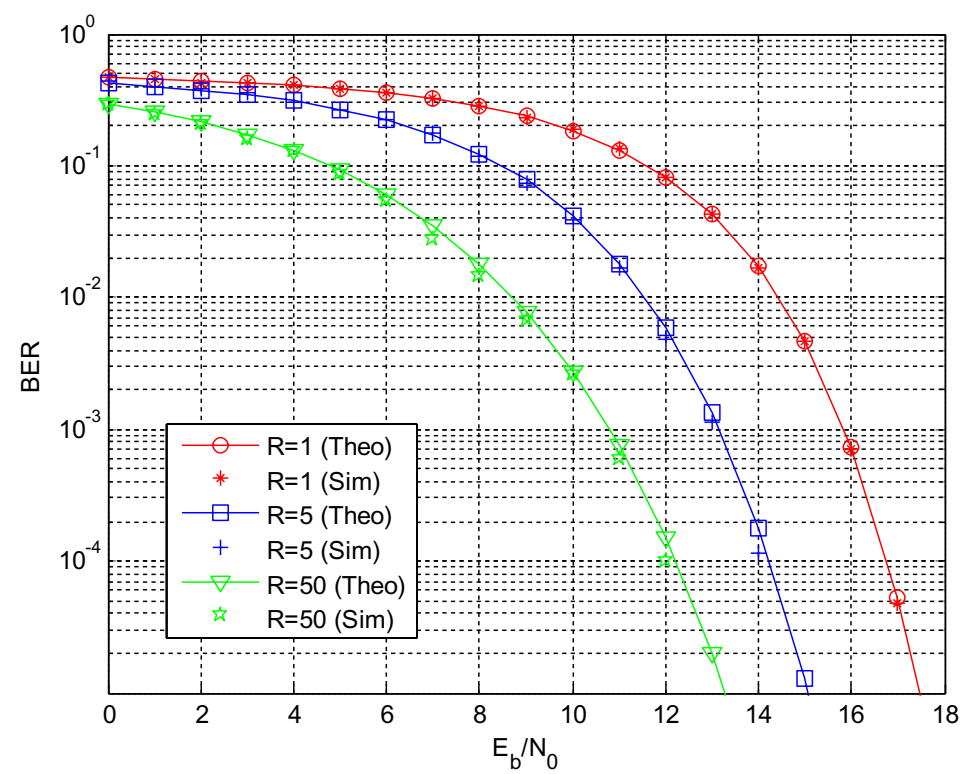

Fig. 4. Analytical and simulated BER performance of NR-MC-DCSK with $\beta=400$ and $M=16$ over AWGN channel.

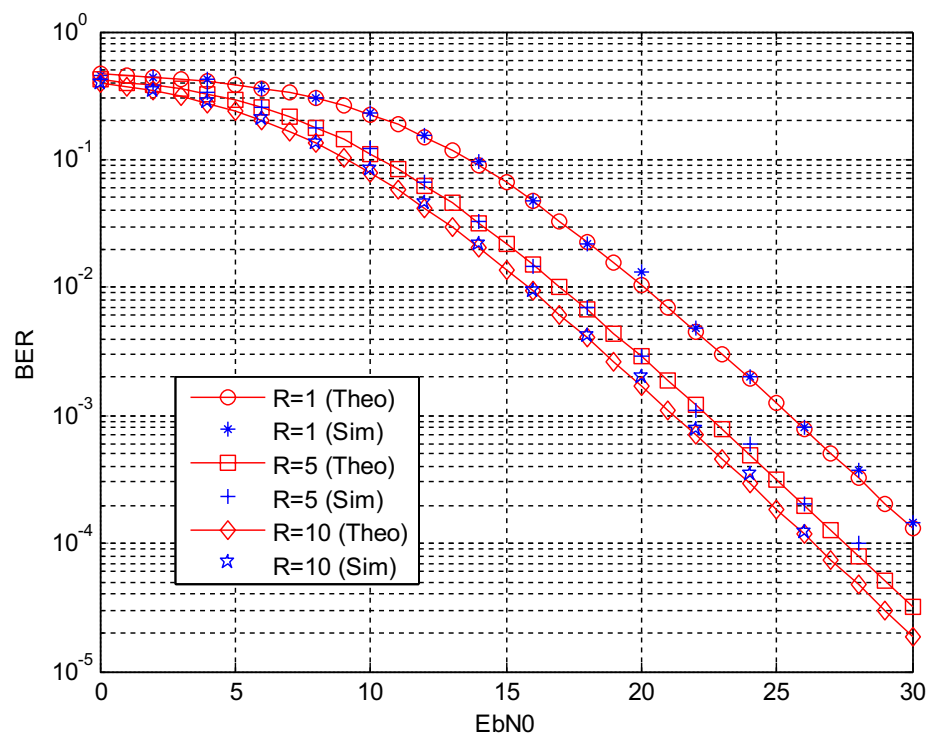

Fig. 5. Analytical and simulated BER performance of NR-MCDCSK with $\beta=400$ and $M=16$ over twopaths Rayleigh fading channel $(L=2)$ with delays: $\lambda_{1}=0, \lambda_{2}=10$ and identical average power gains: $E\left[\alpha_{1}^{2}\right]=E\left[\alpha_{2}^{2}\right]=0.5$. 
H. M. Zhou, Y. Zhang \& Y. Yu

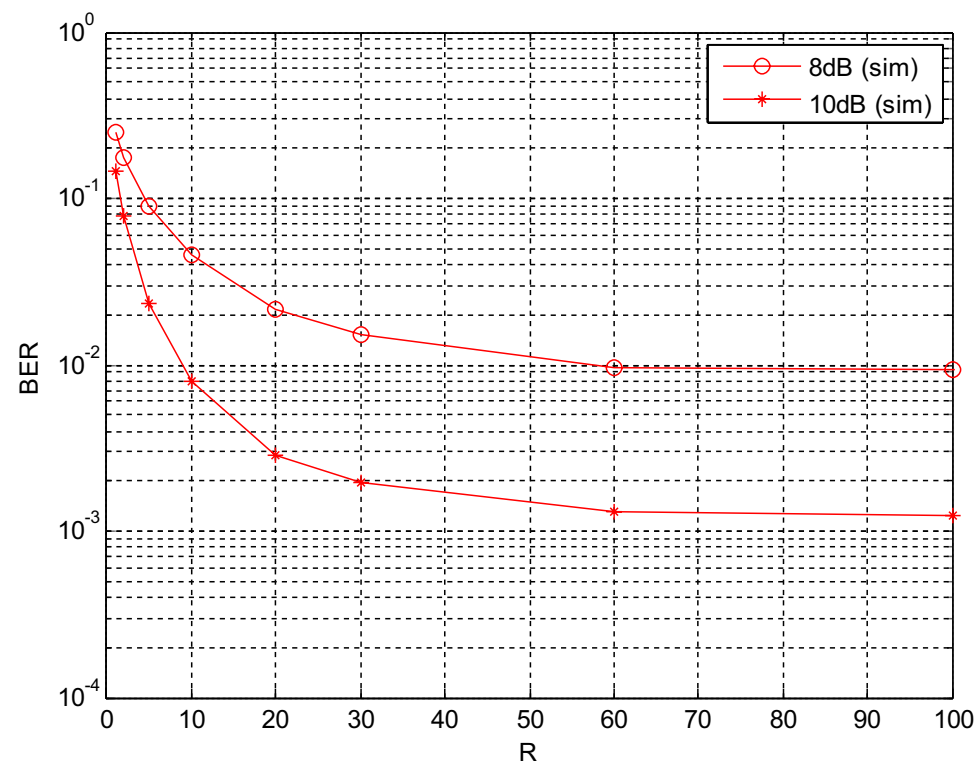

Fig. 6. Relationship between BER and the repeat number $R$ in AWGN channel.

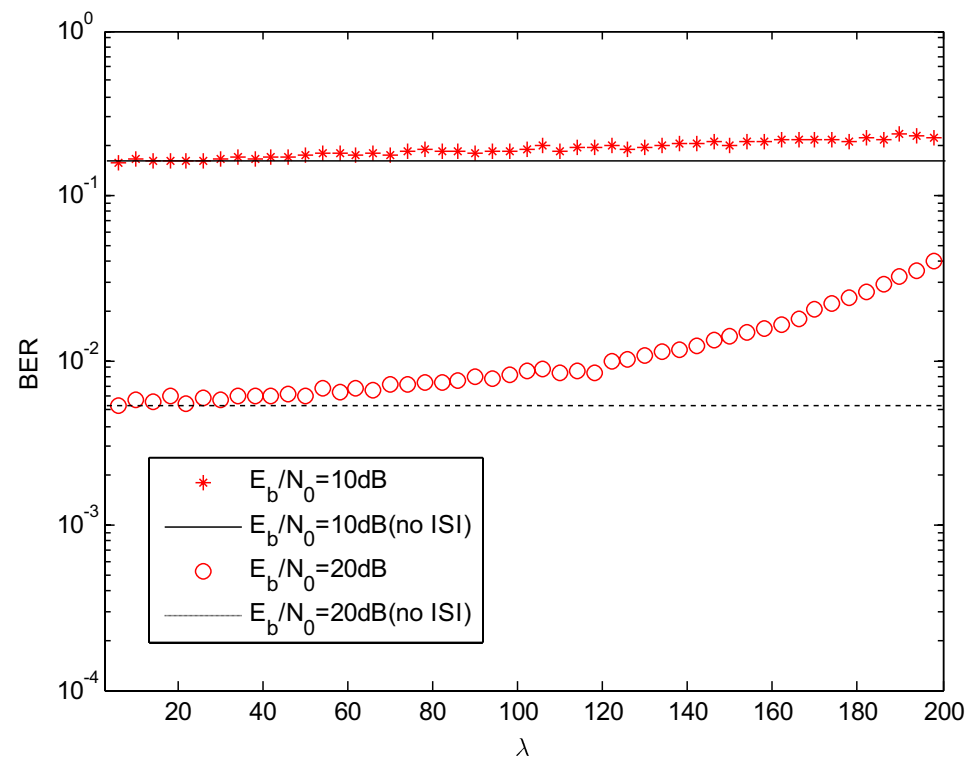

Fig. 7. Influence of time delays on the BER performance of NR-MC-DCSK over two-paths Rayleigh fading channel $(L=2)$ with delays: $\lambda_{1}=0, \lambda_{2}=\lambda$ and identical average power gains: $E\left[\alpha_{1}^{2}\right]=E\left[\alpha_{2}^{2}\right]=0.5$ 


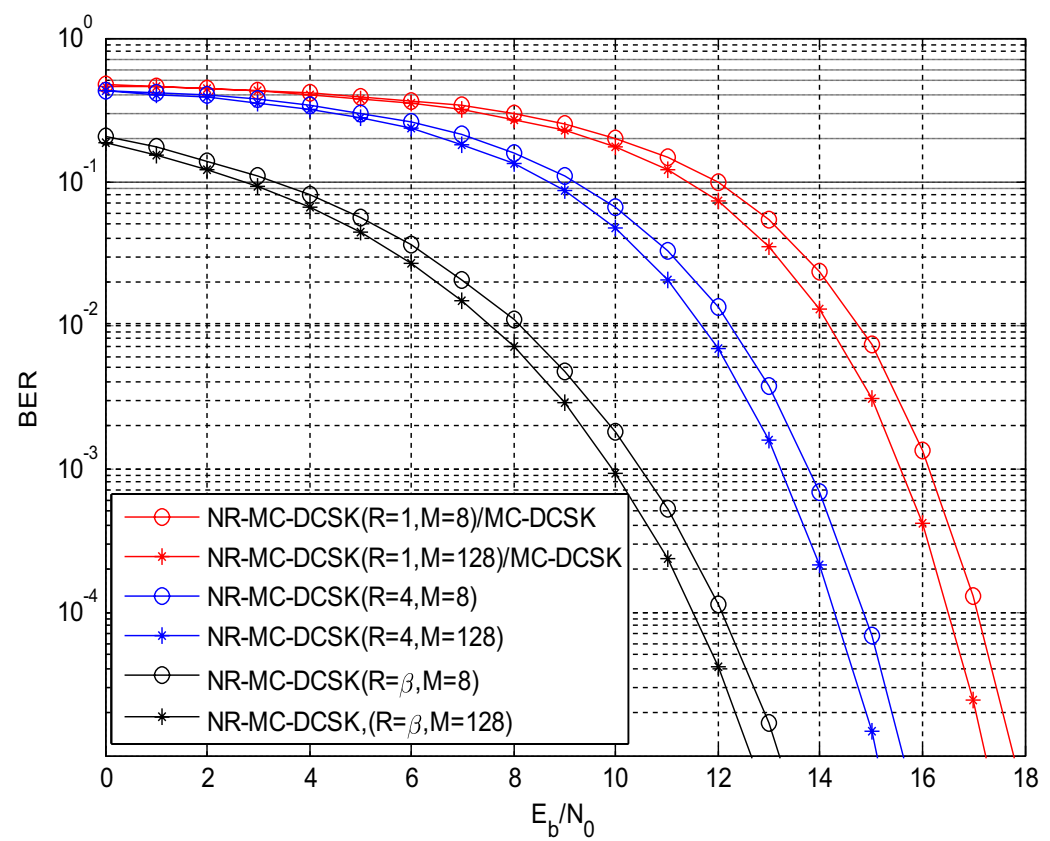

Fig. 8. BER comparison between MC-DCSK and NR-MC-DCSK with a spreading factor $\beta=400$ over AWGN channel.

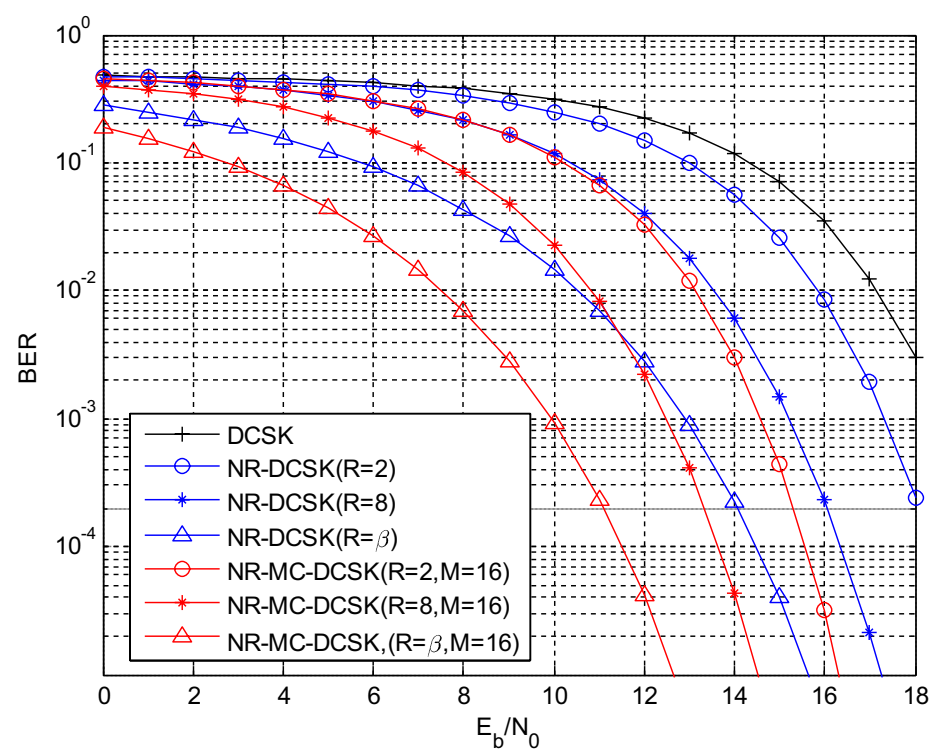

Fig. 9. BER comparison between DCSK, NR-DCSK and NR-MC-DCSK with a spreading factor $\beta=400$ over AWGN channel. 
well. It is also noticed that the increase of time delay deteriorates the performance over multipath fading channels.

The simulated BER performances of NR-MC-DCSK are compared with that of MC-DCSK in Fig. 8 over AWGN channel. In these two systems, the spreading factor is set to 400, and the numbers of subcarriers are set to equal. It is evident that the proposed system performs much better than MC-DCSK system due to reduced noises in decision variables. When $R$ increases, the influence of noises becomes smaller and smaller, thus the BER gain obtained by NR-MC-DCSK grows and eventually reaches the maximal value (up to $5-6 \mathrm{~dB}$ ) when $R=\beta$.

In Fig. 9, the BER performance of the proposed system is also compared to that of DCSK and NR-DCSK systems with same spreading factors. Among these three systems, DCSK performs the worst due to low energy efficiency and the effects of noise interferences. With fewer noises in decision variables, NR-DCSK performs better than DCSK. Compared to DCSK and NR-DCSK, the proposed system shows the best BER performance as it achieves not only noise reduction but also high energy efficiency.

\section{Conclusions}

In this paper, a novel NR-MC-DCSK system that uses duplicated chaotic signals is proposed. In this new system, the reference can be divided into $N$ segments, and each segment is generated by repeating one chaotic sample for $R$ times. As a result, the repetition has been introduced into both the reference and data signals, and noises in received signals can be reduced by averaging all duplicated samples. It is shown that the proposed system obtains same energy efficiency in comparison with MC-DCSK, and it can also outperform MC-DCSK, NR-DCSK and DCSK systems in BER performance. This solid performance enhancement offers NR-MC-DCSK an unprecedented potential in many scenarios where chaotic spread-spectrum communication systems are needed.

\section{Acknowledgments}

This work was supported in part by the National Natural Science Foundation of China under Grant Nos. 61401226 and 61374180, Jiangsu Government Scholarship for Oversea Studies, the University Science Research Project of Jiangsu Province (Grant No. 16KJB510045), and the General Project of Nanjing University of Posts and Telecommunications (Grant No. NY215167).

\section{References}

1. Y. Fang et al., A survey on DCSK-based communication systems and their application to UWB scenarios, IEEE Commun. Surv. Tutor. 18 (2016) 1804-1837. 
2. H. Yang, W. K. S. Tang, G. Chen and G.-P. Jiang, System design and performance analysis of orthogonal multi-level differential chaos shift keying modulation scheme, IEEE Trans. Circuits Syst. I 63 (2016) 146-156.

3. L. Wang, G. Cai and G. Chen, Design and performance analysis of a new multiresolution M-ary differential chaos shift keying communication system, IEEE Trans. Wireless Commun. 14 (2015) 5197-5208.

4. A. Kumar and P. R. Sahu, Performance analysis of differential chaos shift keying modulation with transmit antenna selection, IET Commun. 10 (2016) 327-335.

5. F. Escribano, G. Kaddoum, A. Wagemakers and P. Giard, Design of a new differential chaos-shift-keying system for continuous mobility, IEEE Trans. Commun. 64 (2016) 2066-2078.

6. G. Kolumbán, B. Vizvki, W. Schwarz and A. Abel, Differential chaos shift keying: A robust coding for chaotic communication, Proc. NDES, Seville, Spain (1996), pp. 87-92.

7. G. Kolumbán, G. Kis, M. P. Kennedy and Z. Jáko, FM-DCSK: A new and robust solution to chaos communications, Proc. Int. Symp. Nonlinear Theory Appl., HI (1997), pp. $117-120$.

8. Z. Galias and G. M. Maggio, Quadrature chaos-shift keying: Theory and performance analysis, IEEE Trans. Circuits Syst. I 48 (2001) 1510-1519.

9. M. Sushchik, L. S. Tsimring and A. R. Volkovskii, Performance analysis of correlationbased communication schemes utilizing chaos, IEEE Trans. Circuits Syst. I 47 (2000) $1684-1691$.

10. H. Yang, G.-P. Jiang and J. Duan, Phase-separated DCSK: A simple delay-componentfree solution for chaotic communications, IEEE Trans. Circuits Syst. II 61 (2014) 967-971.

11. W. K. Xu and L. Wang, A novel differential chaos shift keying modulation scheme, Int. J. Bifurcation Chaos 21 (2011) 799-814.

12. G. Kaddoum and F. Gagnon, Design of a high-data-rate differential chaos-shift keying system, IEEE Trans. Circuits Syst. II 59 (2012) 448-452.

13. F. C. M. Lau and C. K. Tse, Chaos-Based Digital Communication Systems (SpringerVerlag, 2003).

14. G. Kaddoum, E. Soujeri and Y. Nijsure, Design of a short reference noncoherent chaosbased communication systems, IEEE Trans. Commun. 64 (2016) 680-689.

15. G. Kaddoum, H.-V. Tran, L. Kong and M. Atallah, Design of simultaneous wireless information and power transfer scheme for short reference DCSK communication systems, IEEE Trans. Commun. 65 (2017) 431-443.

16. G. Kaddoum, F. Richardson and F. Gagnon, Design and analysis of a multi-carrier differential chaos shift keying communication system, IEEE Trans. Commun. 61 (2013) $3281-3291$.

17. G. Kaddoum and F. Shokraneh, Analog network coding for multi-user multi-carrier differential chaos shift keying communication system, IEEE Trans. Wireless Commun. 14 (2015) 1492-1505.

18. G. Kaddoum, Design and performance analysis of a multiuser OFDM based differential chaos shift keying communication system, IEEE Trans. Commun. 64 (2016) 249-260.

19. H. Yang, W. K. S. Tang, G. R. Chen and G. P. Jiang, Multi-carrier chaos shift keying: System design and performance analysis, IEEE Trans. Circuits Syst. I 64 (2017) 2182-2194.

20. G. Kaddoum and E. Soujeri, NR-DCSK: A noise reduction differential chaos shift keying system, IEEE Trans. Circuits Syst. II 63 (2016) 648-652. 
21. G. Kaddoum, P. Charge and D. Roviras, A generalized methodology for bit-error-rate prediction in correlation-based communication schemes using chaos, IEEE Commun. Lett. 13 (2009) 567-569.

22. N. I. Chernov, Limit theorems and Markov approximations for chaotic dynamical systems, Probab. Theory Relat. Fields 101 (1995) 321-362.

23. J. G. Proakis. Digital Communications (McGraw-Hill, 1995).

24. Y. Xia, C. K. Tse and F. C. M. Lau, Performance of differential chaos shift-keying digital communication systems over a multipath fading channel with delay spread, IEEE Trans. Circuits Syst. II 51 (2004) 680-684.

25. M. Dawa, G. Kaddoum and Z. Sattar, A generalized lower bound on the bit-error-rate of DCSK systems over multi-path Rayleigh fading channels, IEEE Trans. Circuits Syst. II 65 (2018) 321-325. 